\title{
Effect of additional valve lesions on left ventricular ejection time in aortic stenosis
}

\author{
PAUL KLIGFIELD ${ }^{1}$, HARVEY GOLDBERG, SUSAN A. KLINE, AND \\ STEPHEN SCHEIDT
}

From The Division of Cardiology, Department of Medicine, Cornell University Medical College, New York, N.Y. 10021, U.S.A.

Rate-corrected left ventricular ejection time was measured from the aortic pressure tracings of 171 catheterised patients with aortic valve area $\leq 1.2 \mathrm{~cm}^{2}$. In 50 patients with pure aortic stenosis, left ventricular ejection time increased with decreasing valve area and was significantly higher ( $468 \pm 5 \mathrm{~ms}$, mean $\pm S E M$ ) than in 13 normal subjects ( $435 \pm 5 \mathrm{~ms})$. Additional aortic regurgitation in 72 patients further increased the left ventricular ejection time to $484 \pm 4 \mathrm{~ms}$.

Significant mitral stenosis (mitral valve area $\leq 1.2 \mathrm{~cm}^{2}$ ) in 6 patients with aortic stenosis and 33 patients with aortic stenosis and regurgitation reduced the left ventricular ejection time to normal. Similarly, severe mitral regurgitation in 3 patients with aortic stenosis and regurgitation reduced left ventricular ejection time to normal, though slight or moderate mitral regurgitation in 7 of these patients did not.

These data show that the prolonged left ventricular ejection time in aortic valve disease $m$ zy be restored to normal in the presence of coexisting significant mitral disease.

Prolongation of the rate-corrected left ventricular ejection time is associated with significant aortic stenosis or aortic regurgitation (Katz and Feil, 1925; Benchimol et al., 1960; Parisi et al., 1971; Bonner et al., 1973; Bache et al., 1973). Though depressed left ventricular function of ischaemic or myopathic aetiology generally shortens the left ventricular ejection time (Weissler et al., 1961; Weissler et al., 1968, 1969; Heikkilä et al., 1971; Pouget et al., 1971; McConahay et al., 1972), it has recently been shown that congestive failure secondary to aortic stenosis can be distinguished from these by persistence of prolongation of left ventricular ejection time (Bonner and Tavel, 1973). Though it is widely appreciated in clinical practice that mitral valvular disease may alter the pulse contour expected in aortic disease, no quantitative study to emphasise and document this effect is available other than the partial data presented by Epstein and Coulshed (1964). Since additional valve lesions commonly exist in the presence of important aortic stenosis, and since mitral valve lesions may be associated with left ventricular ejection time shortening (Benchimol et al., 1960; Moskowitz and Wechsler, 1965; Elkins et al., 1967; Tavel et al., 1972), the

${ }^{1}$ Present address: Naval Submarine Medical Center Groton Conn 06340, U.S.A.

Received for publication 28 February 1977 present study was designed to quantify the effect of additional lesions on the prolonged left ventricular ejection time of aortic stenosis.

\section{Methods}

Left ventricular ejection time was measured from the central aortic pulse tracing obtained at cardiac catheterisation in 171 patients with calculated aortic valve areas of $1.2 \mathrm{~cm}^{2}$ or less, with clear identification of onset of ejection and incisura. Left ventricular ejection time in $\mathrm{ms}$ was measured from the onset of ejection to the incisura and an average value was determined for 4 cycles. Rate correction was performed according to the regression data of Weissler et al. (1969) for patients in sinus rhythm; for men, left ventricular ejection time $=$ measured ejection time $+1.7 \times$ heart rate. For patients with atrial fibrillation, an average value was calculated for cycles of similar length and the average heart rate of these cycles was used for rate correction.

Of the 171 patients, 50 had pure aortic stenosis with valve areas calculated from the Gorlin formula (Gorlin and Gorlin, 1951) (constant $=44.5$ ) from $0.3 \mathrm{~cm}^{2}$ to $1.2 \mathrm{~cm}^{2}$. Rate corrected left ventricular ejection time was compared with calculated orifice size. Mean values of left ventricular ejection time 
for valve areas between $0.3 \mathrm{~cm}^{2}$ and $0.8 \mathrm{~cm}^{2}$ ('severe' aortic stenosis), between $0.9 \mathrm{~cm}^{2}$ and $1.2 \mathrm{~cm}^{2}$ ('moderately severe' aortic stenosis), and for the total group were calculated.

Seventy-two patients had varying degrees of additional aortic regurgitation. This was classified as slight if the aortic root injection disclosed regurgitation of contrast that was cleared from the left ventricle during several beats. Moderate regurgitation was defined as progressive opacification of the ventricle following root injection. Severe regurgitation was defined as ventricular opacification during the one or two beats after root injection. Mean left ventricular ejection time values were calculated for the total aortic stenosis and regurgitation group and also for subgroups arranged according to the degree of aortic stenosis and the amount of aortic regurgitation.

Additional mitral stenosis, with valve areas less than $1.2 \mathrm{~cm}^{2}$ calculated from the Gorlin formula, was found in 3 patients with pure aortic stenosis and 12 patients with combined aortic stenosis and regurgitation. Three patients with aortic stenosis and mitral stenosis had additional slight or moderate mitral regurgitation, and 21 patients with aortic stenosis and regurgitation and mitral stenosis had additional slight mitral regurgitation.

Slight mitral regurgitation was defined as regurgitation of contrast on left ventricular injection that failed to opacify the left atrium. Moderate mitral regurgitation was defined as progressive opacification of the atrium, and severe mitral regurgitation as opacification of the atrium over one or two beats. Seven patients had slight or moderate mitral regurgitation in addition to aortic stenosis and regurgitation. Only 3 patients had severe mitral regurgitation in addition to aortic stenosis and regurgitation, and these had additional slight mitral stenosis, with valve areas greater than $1.2 \mathrm{~cm}^{2}$. There were no patients with significant aortic stenosis and additional pure severe mitral regurgitation.

For comparison, mean left ventricular ejection time was calculated for 13 subjects with normal values and ventricular function, 19 patients with pure isolated mitral stenosis, and 9 patients with pure mitral regurgitation.

\section{Results}

Patient grouping and results are outlined in the Table.

NORMAL PATIENTS

Mean left ventricular ejection time \pm SEM for 13 normal subjects was $435 \pm 5$ ms.
Table

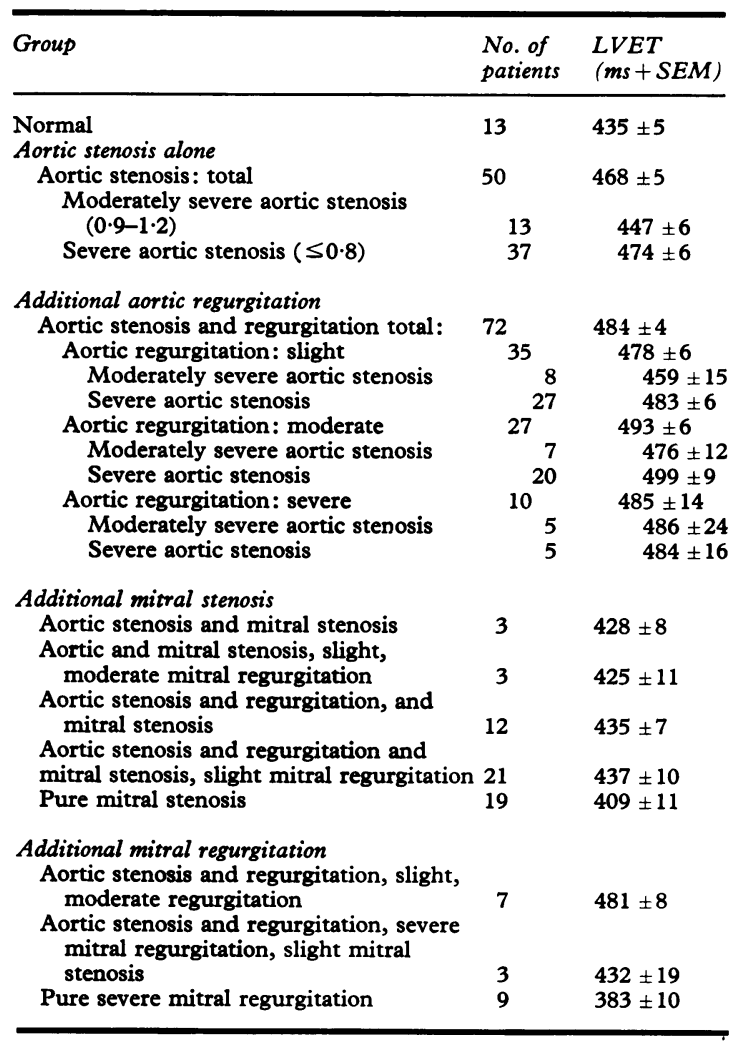

\section{AORTIC STENOSIS}

In the group of patients with pure aortic stenosis, left ventricular ejection time tended to increase with decreasing valve area, as seen in Fig. 1. Regression data relating the variables, left ventricular

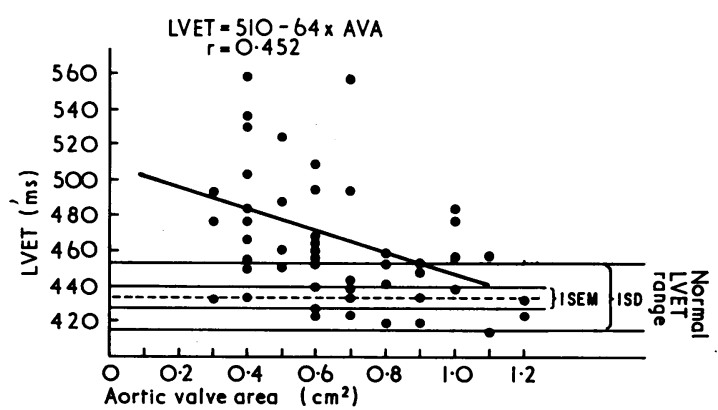

Fig. 1 Left ventricular ejection time (LVET) in 50 patients with calculated aortic valve between 0.3 and $1 \cdot 2 \mathrm{~cm}^{2}$, showing increasing left ventricular ejection time with decreasing orifice size. Linear correlation is poor, and considerable overlap with normal exists for valve areas $>0.6 \mathrm{~cm}^{2}$. 
ejection time $=-64$ aortic valve area +510 , showed wide scatter $(\mathbf{r}=-0 \cdot 45)$. Patients with moderately severe aortic stenosis (valve area 0.9$1.2 \mathrm{~cm}^{2}$ ) had a mean left ventricular ejection time of $447 \pm 6 \mathrm{~ms}$, higher than mean normal, but not significantly $(P<0 \cdot 10)$. Patients with severe aortic stenosis (valve area $\leq 0.8 \mathrm{~cm}^{2}$ ) had a mean left ventricular ejection time of $474 \pm 6 \mathrm{~ms}$, significantly higher than both the normal group and less severe aortic stenosis subgroup $(P<0.001)$. The mean left ventricular ejection time for the entire aortic stenosis group of $468 \pm 5 \mathrm{~ms}$ was significantly higher than normal $(P<0.005)$ (Fig. 2).

\section{ADDITIONAL AORTIC REGURGITATION}

The effect of varying degrees of aortic regurgitation on the left ventricular ejection time in 72 patients with aortic stenosis is shown in Fig. 3. For any calculated valve area, additional aortic regurgitation further increased the left ventricular ejection time. With slight aortic regurgitation, left ventricular ejection time for patients with aortic valve areas $\leq 1.2 \mathrm{~cm}^{2}$ rose to $478 \pm 6 \mathrm{~ms}(P<0.05)$. The increase with moderate aortic regurgitation was more pronounced, with a mean left ventricular ejection time of $493 \pm 7 \mathrm{~ms}(P<0.005)$. With severe aortic regurgitation, the mean left ventricular ejection time of $485 \pm 13 \mathrm{~ms}$ was not significantly higher than the pure aortic stenosis group $(P<0 \cdot 10)$, and was slightly lower than the moderate aortic regurgitation subgroup. For all patients with aortic regurgitation in addition to aortic stenosis, the left ventricular ejection time of $\mathbf{4 8 4} \pm \mathbf{4} \mathrm{ms}$ was significantly greater than both the normal $(P<0.001)$ and pure aortic stenosis $(P<0.01)$ groups.

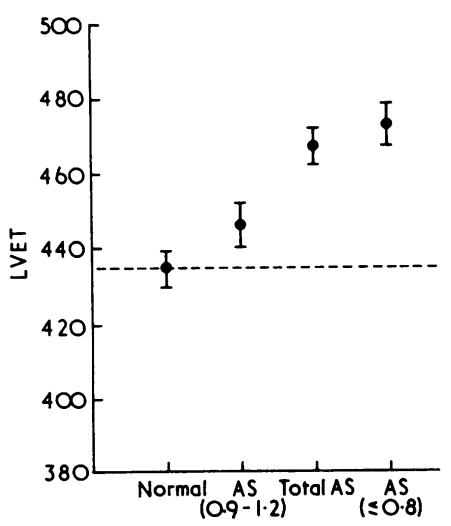

Fig. 2 Mean left ventricular ejection time (LVET) $\pm S E M$ for subgroups of patients with varying severity of pure aortic stenosis (AS). The mean value for the total group is significantly higher than normal.
ADDITIONAL MITRAL STENOSIS

The effect of mitral stenosis on the prolongation of left ventricular ejection time in aortic valve disease is shown in Fig. 4. In 19 patients with pure mitral stenosis (valve area $<1.2 \mathrm{~cm}^{2}$ ) and no aortic disease, the left ventricular ejection time of $409 \pm 17 \mathrm{~ms}$ was significantly lower than the normal, pure aortic stenosis, and aortic stenosis and regurgitation group means $(P<0.001)$. With additional important

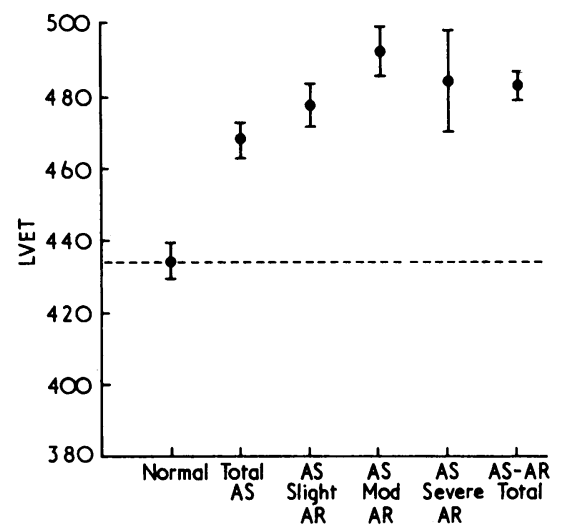

Fig. 3 Effect of varying additional degrees of aortic regurgitation (AR) on the left ventricular ejection time in aortic stenosis ( $A S)$. The mean value for the total aortic stenosis-aortic regurgitation ( $A S-A R$ ) group is significantly higher than both the normal and total aortic stenosis groups.

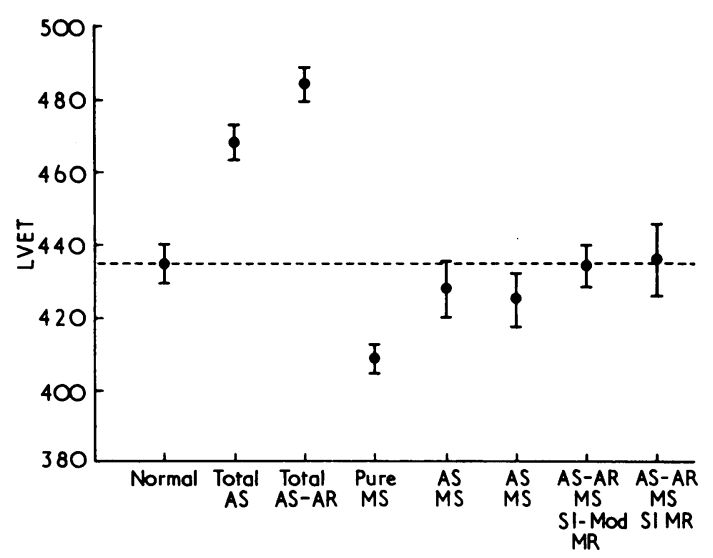

Fig. 4 Effect of additional significant mitral stenosis (MS) on the left ventricular ejection time (LVET). Normalisation of left ventricular ejection time results when mitral stenosis coincides with aortic stenosis ( $A S$ ) or with combined aortc stenosis and regurgitation ( $A S-A R)$. $S l=$ slight $;$ Mod $=$ moderate. 
mitral stenosis, the mean left ventricular ejection time in 3 patients with aortic stenosis of $428 \pm 8 \mathrm{~ms}$ was significantly lower than the left ventricular ejection time in the pure aortic stenosis group $(\mathrm{P}<0.05)$, and not significantly different from normal. In 3 patients with slight or moderate mitral regurgitation in addition to mitral stenosis and aortic stenosis, the mean left ventricular ejection time of $425 \pm 11 \mathrm{~ms}$ was no different from the mitral stenosis and aortic stenosis group and again not different from normal. Similarly, in 12 patients with aortic stenosis and regurgitation additional important mitral stenosis lowered the mean left ventricular ejection time to $435 \pm 7 \mathrm{~ms}$, a value significantly different from the pure aortic stenosis and mitral regurgitation group $(P<0.001)$ and identical to normal. In 21 patients with slight mitral regurgitation in addition to mitral stenosis, aortic stenosis, and regurgitation, the mean left ventricular ejection time of $437 \pm 10 \mathrm{~ms}$ was not significantly different from the mean left ventricular ejection time value of the important lesions together or from normal.

\section{ADDITIONAL MITRAL REGURGITATION}

The effect of additional mitral regurgitation on left ventricular ejection time prolongation in aortic valve disease is shown in Fig. 5. In 9 patients with pure severe mitral regurgitation and no aortic valve disease, the mean left ventricular ejection time of $383 \pm 10 \mathrm{~ms}$ was significantly lower than normal, pure aortic stenosis, and aortic stenosis and regurgi-

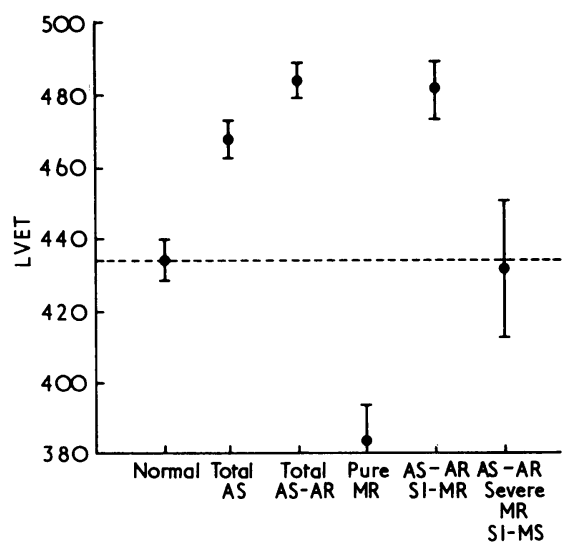

Fig. 5 Effect of additional mitral regurgitation. Return to normal cf left ventricular ejection time (LVET) results when severe mitral regurgitation (MR) coincides with aortic stenosis and regurgitation $(A S-A R)$, but this effect is not seen with lesser degrees of mitral regurgitation (MR). Sl, slight. tation group means $(P<0.001)$. Additional slight or moderate mitral regurgitation in 7 patients with aortic stenosis and regurgitation insignificantly lowered the mean left ventricular ejection time to $481 \pm 8 \mathrm{~ms}$ compared with the pure aortic stenosis and regurgitation group, and this value remained significantly higher than normal $(P<0.001)$. In 3 patients with severe mitral regurgitation, however, who had additional slight mitral stenosis (valve area $>1.2 \mathrm{~cm}^{2}$ ) and aortic stenosis and regurgitation, the mean left ventricular ejection time of $432 \pm 10 \mathrm{~ms}$ was significantly lower than the pure aortic stenosis and regurgitation group $(P<0.01)$ but not significantly different from normal.

\section{Discussion}

The results of this study indicate that significant mitral stenosis and severe mitral regurgitation may return the left ventricular ejection time to normal in patients with aortic stenosis. Previous work has emphasised the clinical usefulness of left ventricular ejection time prolongation caused by outflow obstruction or high stroke volume in identifying patients with aortic stenosis or aortic regurgitation (Katz and Feil, 1925; Benchimol et al., 1960; Parisi et al., 1971; Bache et al., 1973; Bonner et al., 1973). It has also been shown that this prolongation is maintained in the presence of left ventricular failure caused by aortic stenosis (Bonner and Tavel, 1973), allowing separation of this important group to be made from patients with ventricular dysfunction of ischaemic, myopathic, and hypertensive aetiologies in which left ventricular ejection time is shortened (Weissler et al., 1961; Weissler et al., 1968, 1969; Heikkilä et al., 1971; Pouget et al., 1971; McConahay et al., 1972).

Pure mitral stenosis is usually associated with a shortened left ventricular ejection time (Benchimol et al., 1960; Moskowitz and Wechsler, 1965; Tavel et al., 1972), depending in part on preceding cycle length for patients in atrial fibrillation (Kligfield, 1974), since left ventricular filling and subsequent ejection are limited by inflow obstruction and diastolic filling time. This was seen in our 19 patients with pure mitral stenosis.

In patients with both aortic and mitral lesions, our data confirm and extend the work of Epstein and Coulshed (1964), who showed progressive shortening of ejection time in aortic stenosis with increasing severity of mitral stenosis. However, the degree of aortic stenosis in their patients was not specified, and ejection times were corrected for heart rate by the Bazett formula rather than from regression data. In our patients, significant mitral stenosis 
reduced the left ventricular ejection time to normal in patients with aortic stenosis or combined aortic stenosis and regurgitation.

Pure severe mitral regurgitation is generally associated with a shortened left ventricular ejection time (Moskowitz and Wechsler, 1965; Elkins et al., 1967), as seen in our 9 cases. With this lesion, abbreviation of ejection results from failure of the left ventricle to maintain forward flow during late systole (Elkins et al., 1967). Other studies have reported normal duration of ejection in mitral regurgitation (Wiggers and Feil, 1921-1922; Nixon and Wagner, 1962), or were not rate corrected (Nixon and Wagner, 1962). It would be expected that lesser degrees of regurgitation would have less shortening effect on the left ventricular ejection time. This would account for the persistence of left ventricular ejection time prolongation in patients with aortic stenosis and regurgitation with only slight or moderate mitral regurgitation in this series compared with shortening to normal with additional severe regurgitation. In patients with combined aortic and mitral lesions (both mitral stenosis and regurgitation) the reduction of left ventricular ejection time to normal was not the result of left ventricular failure; there was no correlation between left ventricular ejection time and left ventricular end-diastolic pressure in patients with combined valvular lesions.

Use of the Gorlin formula tends to underestimate aortic valve area in the presence of aortic regurgitation, since calculated forward flow is less than the true cardiac output. Because of this, the degree of aortic stenosis in the patient groups with increasing regurgitation is correspondingly less than calculated. Milder stenosis may, therefore, account for the slight decrease in mean left ventricular ejection time in the aortic stenosis-severe aortic regurgitation group compared with the aortic stenosis-moderate regurgitation group. Alternatively, depressed ventricular function in the patients with severe aortic regurgitation may shorten the left ventricular ejection time, though there was no correlation between left ventricular end-diastolic pressure and left ventricular ejection time or between left ventricular end-diastolic pressure and aortic valve area in the $\mathbf{1 0}$ patients with aortic stenosis and severe aortic regurgitation. Whatever the mechanism, however, the difference from normal left ventricular ejection time at all levels of additional aortic regurgitation remains highly significant.

The effect of additional valve lesions on the duration of ejection in aortic stenosis therefore appears to be additive. Since mitral and aortic valve lesions frequently coexist, it is important to recognise that significant mitral disease can mask left ventricular ejection time prolongation, and conversely, that a normal left ventricular ejection time does not exclude severe mixed aortic and mitral disease. This is consistent with the wellrecognised obscuring of physical signs that occurs in combined valvular disease (Katznelson et al., 1960; Honey, 1961; Morrow et al., 1962; Reid et al., 1962; Zitnik et al., 1965).

The duration of ejection measured from the central aortic pressure pulse has been shown to correlate closely with the left ventricular ejection time obtained non-invasively from external carotid pulse recording (Weissler et al., 1961; Martin et al., 1971). These results are, therefore, applicable to interpretation of standard systolic time intervals as popularised by Weissler et al. (1961, 1968). The regression data of Weissler et al. (1969) were used for rate correction in our patients, since these equations have become the standard for most investigators. Independently derived regression data for rate correction in our patients and in other series differ slightly from the equation used, but use of such data did not change the significance of the results presented. Though the direction of changes reported in this study is valid, each laboratory must establish its own range of normal before comparisons can be made.

It must also be appreciated that while valvular dysfunction affects the duration of ejection, independent variations of preload, contractile state, and afterload may alter left ventricular ejection time (Braunwald et al., 1958; Weissler et al., 1961; Heikkilä et al., 1971; Martin et al., 1971). This probably accounts for the wide range of values seen within each subgroup studied, so that even where mean values between groups are highly significantly different, some overlap of individual patient values generally occurs. This effect is well seen in the comparison of left ventricular ejection time and calculated valve area for patients with pure aortic stenosis. Only at valve areas below $0.6 \mathrm{~cm}^{2}$ do the majority of left ventricular ejection time values not overlap the normal range, even though the mean left ventricular ejection time associated with valve areas below $0.9 \mathrm{~cm}^{2}$ is highly significantly different from normal.

The poor correlation $(r=0.45)$ between left ventricular ejection time and calculated aortic valve orifice area is similar to that found by Bache et al. (1973), though these workers were able to improve prediction of valve area with prolongation of ejection above a value obtained from stroke volume regression data. In the past, accurate stroke volume determination generally required intravascular measurement, so this correlation was of limited clinical usefulness. As noninvasive determination of 
stroke volume improves, for instance using ultrasound or radionuclide imaging, addition of stroke volume regression equations to carotid pulse left ventricular ejection time measurements may greatly improve indirect estimation of aortic valve orifice area.

\section{References}

Bache, R. J., Wang, Y., and Greenfield, J. C., Jr. (1973). Left ventricular ejection time in valvular aortic stenosis. Circulation, 47, 527-533.

Benchimol, A., Dimond, E. G., and Shen, Y. (1960). Ejection time in aortic stenosis and mitral stenosis: comparison between the direct and indirect arterial tracings, with special reference to pre- and postoperative findings. American fournal of Cardiology, 5, 728-743.

Bonner, A. J., Sacks, H. N., and Tavel, M. E. (1973). Assessing the severity of aortic stenosis by phonocardiography and external carotid pulse recordings. Circulation, 48, 247252.

Bonner, A. J., and Tavel, M. E. (1973). Systolic time intervals: use in congestive heart failure due to aortic stenosis. Archives of Internal Medicine, 132, 816-819.

Braunwald, E., Sarnoff, S. J., and Stainsby, W. N. (1958). Determinants of duration and mean rate of ventricular ejection. Circulation Research, 6, 319-325.

Elkins, R. C., Morrow, A. G., Vasko, J. S., and Braunwald, E. (1967). The effects of mitral regurgitation on the pattern of instantaneous aortic blood flow: clinical and experimental observations. Circulation, 36, 45-53.

Epstein, E. J., and Coulshed, N. (1964). Assessment of aortic stenosis from the external carotid pulse wave. British Heart fournal, 26, 84-96.

Gorlin, R., and Gorlin, S. G. (1951). Hydraulic formula for calculation of the area of the stenotic mitral valve, other cardiac valves, and central circulatory shunts. I. American Heart fournal, 41, 1-29.

Heikkilä, J., Luomanmäki, K., and Pyörälä, K. (1971). Serial observations on left ventricular dysfunction in acute myocardial infarction. II: Systolic time intervals in power failure. Circulation, 44, 343-354.

Honey, M. (1961). Clinical and haemodynamic observations on combined mitral and aortic stenosis. British Heart Fournal, 23, 545-555.

Katz, L. N., and Feil, H. S. (1925). Clinical observations on the dynamics of ventricular systole. III-Aortic stenosis and aortic insufficiency. Heart, 12, 171-180.

Katznelson, G., Jreissaty, R. M., Levinson, G. E., Stein, S. W., and Abelmann, W. A. (1960). Combined aortic and mitral stenosis: a clinical and physiological study. American Fournal of Medicine, 29, 242-256.
Kligfield, P. (1974). Systolic time intervals in atrial fibrillation and mitral stenosis. British Heart fournal, 36, 798-805.

McConahay, D. R., Martin, C. M., and Cheitlin, M. D. (1972). Resting and exercise systolic time intervals: correlations with ventricular performance in patients with coronary artery disease. Circulation, 45, 592-601.

Martin, C. E., Shaver, J. A., Thompson, M. E., Reddy, P. S., and Leonard, J. J. (1971). Direct correlation of external systolic time intervals with internal indices of left ventricular function in man. Circulation, 44, 419-431.

Morrow, A. G., Awe, W. C., and Braunwald, E. (1962). Combined mitral and aortic stenosis. British Heart fournal, 24, 606-612.

Moskowitz, R. L., and Wechsler, B. M. (1965). Left ventricular ejection time in aortic and mitral valve disease. American fournal of Cardiology, 15, 809-814.

Nixon, P. G. F., and Wagner, G. R. (1962). The duration of left ventricular systole in mitral incompetence. British Heart fournal, 24, 464-468.

Parisi, A. F., Salzman, S. H., and Schechter, E. (1971). Systolic time intervals in severe aortic valve disease. Circulation, 44, 539-547.

Pouget, J. M., Harris, W. S., Mayron, B. R., and Naughton, J. P. (1971). Abnormal responses of the systolic time intervals to exercise in patients with angina pectoris. Circulation, 43, 289-298.

Reid, J. M., Stevenson, J. G., Barclay, R. S., and Welsh, T. M. (1962). Combined aortic and mitral stenosis. British Heart fournal, 24, 509-515.

Tavel, M. E., Baugh, D. O., Feigenbaum, H., and Nasser, W. K. (1972). Left ventricular ejection time in atrial fibrillation. Circulation, 46, 744-752.

Weissler, A. M., Harris, W. S., and Schoenfeld, C. D. (1968). Systolic time intervals in heart failure in man. Circulation, 37, 149-159.

Weissler, A. M., Harris, W. S., and Schoenfeld, C. D. (1969). Bedside technics for the evaluation of ventricular function in man. American fournal of Cardiology, 23, 577-583.

Weissler, A. M., Peeler, R. G., and Roehll, W. H. (1961). Relationships between left ventricular ejection time, stroke volume and heart rate in normal individuals and patients with cardiovascular disease. American Heart fournal, 62, 367-378.

Wiggers, C. J., and Feil, H. (1921-1922). The cardiodynamics of mitral insufficiency. Heart, 9, 149-174.

Zitnik, R. S., Piemme, T. E., Messer, R. J., Reed, D. P., Hayes, F. W., and Dexter, L. (1965). The masking of aortic stenosis by mitral stenosis. American Heart fournal, 69, 22-30.

Requests for reprints to Dr. Stephen Scheidt, The New York Hospital-Cornell Medical Center, 525 East 68th Street, New York, N.Y. 10021 , U.S.A. 